\title{
The Estimates of Periodic Potentials in Terms of Effective Masses
}

\section{E. Korotyaev ${ }^{\star}$}

Math. Dept. 2, ETU, 5 Prof. Popov Str., St. Petersburg, 197376, Russia. E-mail: evgeni@niif.spb.su

Received: 27 February 1996/Accepted: 27 June 1996

Abstract: Let $G_{n}=\left(A_{n}^{-}, A_{n}^{+}\right), n \geqq 1$, denote the gaps, $M_{n}^{ \pm}$be the effective masses and $\Sigma_{n}=\left[A_{n-1}^{+}, A_{n}^{-}\right], A_{0}^{+}=0$, be the spectral bands of the Hill operator $T=$ $-d^{2} / d x^{2}+V(x)$ in $L^{2}(\mathbf{R})$, where $V$ is a 1-periodic real potential from $L^{2}(0,1)$. Let the length gap $L_{n}=\left|G_{n}\right|, h_{n}$ be the height of the corresponding slit on the quasimomentum domain and $\Delta_{n}=\pi^{2}(2 n-1)-\left|\Sigma_{n}\right|>0$ be the band reduction. Let $l_{n}=\sqrt{A_{n}^{+}}-\sqrt{A_{n}^{-}}, n \geqq 1$, denote the gap length for the operator $\sqrt{T} \geqq 0$. Introduce the sequences $L=\left\{L_{n}\right\}, h=\left\{h_{n}\right\}, l=\left\{l_{n}\right\}, \Delta=\left\{\Delta_{n}\right\}, M^{ \pm}=\left\{M_{n}^{ \pm}\right\}$and the norms $\|f\|_{m}^{2}=\sum_{n>0}(2 \pi n)^{2 m} f_{n}^{2}, m \geqq 0$. The following results are obtained: i) The estimates of $\|V\|,\|L\|,\|h\|_{1},\|l\|_{1},\|\Delta\|$ in terms of $\left\|M^{ \pm}\right\|_{2}$, ii) identities for the Dirichlet integral of quasimomentum and integral of potentials and so on, iii) the generation of i), ii) for more general potentials.

\section{Introduction}

Let us consider the Hill operator $T=-d^{2} / d x^{2}+V(x)$ in $L^{2}(\mathbf{R})$, where $V$ is a 1-periodic real potential from $L^{1}(0,1)$. It is well known that the spectrum of $T$ is absolutely continuous and consists of intervals $\Sigma_{1}, \Sigma_{2}, \ldots$. Here $\Sigma_{n}=$ $\left[A_{n-1}^{+}, A_{n}^{-}\right], \ldots, A_{n-1}^{+}<A_{n}^{-} \leqq A_{n}^{+}, n \geqq 1$, and let $A_{0}^{+}=0$. These intervals are separated by the gaps $G_{1}, G_{2}, \ldots$, where $G_{n}=\left(A_{n}^{-}, A_{n}^{+}\right)$. If a gap degenerates, i.e. $G_{n}=\emptyset$, then the corresponding segments $\Sigma_{n}, \Sigma_{n+1}$ merge. Let $\varphi(x, E), \vartheta(x, E)$ be the solutions of the equation

$$
-f^{\prime \prime}+V f=E f, \quad E \in \mathbf{C},
$$

satisfying $\varphi^{\prime}(0, E)=\vartheta(0, E)=1$ and $\varphi(0, E)=\vartheta^{\prime}(0, E)=0$. We define the Lyapunov function $F(E)=\left(\varphi^{\prime}(1, E)+\vartheta(1, E)\right) / 2$. The sequence $A_{0}^{+}<A_{1}^{-} \leqq A_{1}^{+}<\cdots$ is the spectrum of Eq. (1.1) with the periodic boundary conditions of period 2, i.e. $f(x+2)=f(x), x \in \mathbf{R}$. Here the equality means that $A_{n}^{-}=A_{n}^{+}$is the double eigenvalue. We note that $F\left(A_{n}^{ \pm}\right)=(-1)^{n}, n \geqq 1$. The lowest eigenvalue $A_{0}^{+}$is simple, $F\left(A_{0}^{+}\right)=1$, and the corresponding eigenfunction has period 1 . The eigenfunctions

$\star$ The research described in this publication was made possible in part by grant from the Russian Fund of Fundamental Research and INTAS. 
corresponding to $A_{n}^{ \pm}$have period 1 when $n$ is even and they are antiperiodic, $f(x+1)$ $=-f(x), x \in \mathbf{R}$, when $n$ is odd. Define a quasimomentum function (see [F,MO])

$$
k(w)=\arccos F\left(w^{2}\right), \quad w \in W=\mathbf{C} \backslash \bigcup g_{n} .
$$

Here $g_{n}=\left(a_{n}^{-}, a_{n}^{+}\right)=-g_{n}, n \geqq 1$, and $a_{n}^{ \pm}=\sqrt{A_{n}^{ \pm}}>0$. The function $k(w)$ is analytic and moreover $k(w)$ is a conformal mapping from $W$ onto a quasimomentum domain $K=\mathbf{C} \backslash \bigcup \gamma_{n}$, where $\gamma_{n}=\left(\pi n+i h_{n}, \pi n-i h_{n}\right)$ is an excised slit with the height $h_{n}=h_{-n} \geqq 0, n \in \mathbf{Z}$, and $h_{0}=0$. Any non-degenerate (degenerate) slit $\gamma_{ \pm n}$ is connected in some way with the non-degenerate (degenerate) gap $g_{ \pm n}$ and the non-degenerate (degenerate) energy gap $G_{n}$. Let $w(k)$ be the inverse function for $k(w)$ and $E(k)=w(k)^{2}$. With an edge of the gap $G_{n}$ with the length $L_{n}$ we associate the effective masses $M_{n}^{ \pm}=0$ if $L_{n}=0$ and $M_{n}^{ \pm}=1 / E^{\prime \prime}\left(k\left(a_{n}^{ \pm}\right)\right)$if $L_{n} \neq 0$ and let $M_{0}=1 / E^{\prime \prime}(0)$ be the effective mass for the point zero. It is well known that if $L_{n} \neq 0$, then $\pm M_{n}^{ \pm}>0$ and moreover

$$
E(k)=A_{n}^{ \pm}+\frac{(k-\pi n)^{2}}{2 M_{n}^{ \pm}}(1+o(1)) \quad \text { as } E \rightarrow A_{n}^{ \pm} .
$$

We rewrite a potential $V$ in the form $V=V_{0}+V_{1}$, where $V_{0}=\int_{0}^{1} V(x) d x$. The value $V_{0}$ is an important parameter for the Hill operator. For example for the Hill operator $T_{1}=-d^{2} / d x^{2}+V_{1}(x)$ in $L^{2}(\mathbf{R})$ the value $V_{0}$ is the distance between the zero and the beginning of the spectrum of $T_{1}$.

For a potential $V$ we introduce the sequences $L=\left\{L_{n}\right\}_{1}^{\infty}, h=\left\{h_{n}\right\}_{1}^{\infty}, l=$ $\left\{l_{n}\right\}_{1}^{\infty}$, where $l_{n}=\left|g_{n}\right|$. If a potential $V=0$ then the corresponding spectral band $\Sigma_{n}^{0}=\left[\pi^{2}(n-1)^{2}, \pi^{2} n^{2}\right], n>0$, with the band length $\left|\Sigma_{n}^{0}\right|=\pi^{2}(2 n-1)$. It is well known that if $V \neq 0$, then $\left|\Sigma_{n}^{0}\right|>\left|\Sigma_{n}\right|$, i.e. the band lengths are contracted [Mos]. We introduce the band reduction $\Delta_{n}=\left|\Sigma_{n}^{0}\right|-\left|\Sigma_{n}\right|$, the sequence $\Delta=\left\{\Delta_{n}\right\}_{1}^{\infty}$, the real Hilbert spaces $L_{R}^{2}(0,1)$ with the norm $\|V\|^{2}=\int_{0}^{1} V(x)^{2} d x$ and

$$
\ell_{m}^{2}=\left\{f=\left\{f_{n}\right\}_{1}^{\infty},\|f\|_{m}<\infty\right\}
$$

with the norm

$$
\|f\|_{m}^{2}=\sum_{n>0}(2 \pi n)^{2 m} f_{n}^{2}, \quad m \geqq 0, \ell_{0}^{2}=\ell^{2} .
$$

We define the maps $V \rightarrow h(V)=\left\{h_{n}\right\}, V \rightarrow l(V)=\left\{l_{n}\right\}, V \rightarrow M(V)=\left\{M_{n}\right\}, V \rightarrow$ $L(V)=\left\{L_{n}\right\}, V \rightarrow \Delta(V)=\left\{\Delta_{n}\right\}$.

Let us describe the main results of the present paper.

i) Estimates $\|V\|,\|L\|,\|l\|_{1},\|h\|_{1},\|\Delta\|$ in terms of $\left\|M^{ \pm}\right\|_{2}$ are obtained. obtained.

ii) Estimates $h_{n}, L_{n}$ in terms of the effective mass $M_{n}^{ \pm}$at fixed $n \geqq 1$ are

iii) New identities are found.

iv) There is the extension of i)-iii) for other cases (finite band potentials, limit periodic potentials, etc.).

The estimates of type i) are important for the inverse problems of the Hill operator. First estimates $\left(\|V\|\right.$ in terms of $\left.\|h\|_{1}\right)$ were obtained in the paper [MO] and in the book [M]. In the paper [GT2] (devoted to the inverse problem $V \rightarrow L(V)=\left\{L_{n}\right\}$, by the direct method) the needed estimates $\left\|V_{1}\right\|$ in terms of $\|L\|$ were absent. Some estimates were found in the papers [KK1, KK2]. In 
[KK2] the inverse problems by the direct method for the mappings $V \rightarrow h, V \rightarrow l$, $V \rightarrow L, V \rightarrow M^{ \pm}$were studied. The needed estimates for $\|h\|_{1},\|l\|_{1},\|L\|,\left\|M^{ \pm}\right\|_{2}$ in terms of $\left\|V_{1}\right\|$ and an inverse were obtained, with the exception of two estimates $\left\|V_{1}\right\|$ both in terms of $\left\|M^{ \pm}\right\|_{2}$ and $\|L\|$. The various (and more precise) estimates in terms of $\left\|V_{1}\right\|$ and an inverse were obtained in the paper [K3] both for periodic potentials and for more general ones. For example, the following doublesided estimates hold: $\max \left\{\|L\|,\|l\|_{1},\|h\|_{1}\right\} \leqq 6\left\|V_{1}\right\|\left(1+\left\|V_{1}\right\|^{1 / 3}\right)$ and an inverse $\left\|V_{1}\right\| \leqq 20 \omega(1+\omega)$, where $\omega=\min \left\{\|L\|,\|l\|_{1},\|h\|_{1}\right\}$. In [K4] the author obtains similar estimates for the Dirac operator. There are a lot of identities associated with the Hill operator, for example, the very useful identity is obtained in the paper [MO]:

$$
\int_{R} u^{2} \operatorname{Im} k(u) d u / \pi=\int_{0}^{1} V^{2}(x) d x / 8, \quad w=u+i v, \quad k=p+i q .
$$

Some identities were found in the papers [KK1, K2]. A few new identities are found in the present paper and the more interesting identities are presented in Theorem 2.3 (see (2.12)). This result is very useful for the Hill operator since we have the equalities for the norm of $V_{1}$, the Dirichlet integral, some moments and so on. The possibilities of these identities are shown in [K3], where the estimates of $\|L\|,\|h\|_{1},\|l\|_{1}$ in terms of $\int_{0}^{1} V(x)_{1}^{2} d x$ and an inverse are obtained.

Let us shortly describe the proof. In order to prove i)-iv) we use and study conformal mappings ("effective energy") $r(E)=k(\sqrt{E})^{2}, E \in \mathbf{C}_{+}$, where $k$ is the quasimomentum of the Hill operator. The function $r(E)$ has the analytical continuation on the domain $\mathbf{E}=\mathbf{C} \backslash \bigcup G_{n}$. It is important that the function $r$ is the conformal mapping from $\mathbf{E}$ onto the domain $\mathscr{R}=\mathbf{C} \backslash \Gamma$, where $\Gamma=\bigcup \Gamma_{n}$ and a parabolic slit $\Gamma_{n}=\left\{r=(\pi n+i q)^{2},-h_{n}<q<h_{n}\right\}, n \geqq 1$. That makes it possible to reformulate the problems for the differential operator as the problems of the conformal mapping theory. Then we should study the geometry properties of the conformal mapping from $\mathbf{E}$ onto a "parabolic comb" $\mathscr{R}$. In this case we use the methods, estimates from $[\mathrm{KK} 1, \mathrm{~K} 1]$ and the identities from [K2]. First we shall get local estimates in Sect. 3. Then we obtain some identities and inequalities for the effective energy in Sect. 4. In the last Sect. 5 we shall get the main estimates for the conformal mappings and the main results for the Hill operator.

\section{The Main Results}

In this section we introduce the concepts and the facts needed to formulate the theorems and recall some results for the Hill operator. Recall that the potential $V$ has the form $V=V_{0}+V_{1}$, where $\int_{0}^{1} V_{1}(x) d x=0$ and $V_{0} \geqq 0$ is a constant since $A_{0}^{+}=0$. We introduce the sequence $M=\left\{M_{n}\right\}_{1}^{\infty}$, where $M_{n}=\min \left|M_{n}^{ \pm}\right|$. We present the main estimates on $L, h, V, \Delta$.

Theorem 2.1. Let $V \in L^{2}(0,1)$. Then the following estimates:

$$
\begin{gathered}
\|h\|_{1} \leqq 72 \pi M_{0}\|M\|_{2}, \\
\left\|V_{1}\right\| \leqq \frac{8}{\pi} M_{0}^{1 / 2}\|h\|_{1} \leqq(24)^{2} M_{0}^{3 / 2}\|M\|_{2},
\end{gathered}
$$




$$
\begin{gathered}
\|L\| \leqq \frac{16}{\pi} M_{0}\|h\|_{1} \leqq 2(24)^{2} M_{0}^{2}\|M\|_{2}, \\
\|V\| \leqq \sqrt{2}(24)^{2} M_{0}^{2}\|M\|_{2}, \\
\|\Delta\| \leqq(72)^{2} M_{0}^{2}\|M\|_{2},
\end{gathered}
$$

hold.

Let us note that by (2.10) we have $M_{0}-1 / 2=-\sum_{n>0}\left(M_{n}^{+}+M_{n}^{-}\right)$. Then $M_{0}-$ $1 / 2 \leqq(1 / \sqrt{6})\left\|M^{+}+M^{-}\right\|_{1}$, since $\sum_{n>0} n^{-2}=\pi^{2} / 6$. Hence by $(2.1-5)$ we estimate $\|\Delta\|,\left\|V_{1}\right\|,\|L\|,\|h\|_{1}$ in terms of $\left\|M^{ \pm}\right\|_{2}$. Remark that these estimates are nonlinear. Now we present the main "linear" and local estimates. We define the sequence $M^{+}-M^{-}=\left\{M_{n}^{+}-M_{n}^{-}\right\}_{1}^{\infty}$, where $M_{n}^{+}-M_{n}^{-} \geqq 0$ for all $n \geqq 1$.

Theorem 2.2. Let $V \in L^{2}(0,1)$. Then the following estimates:

$$
\begin{gathered}
\max \left\{\|\Delta\|,\left\|V_{1}\right\|,\|L\|,\|h\|_{1}\right\} \leqq 8 \pi\left\|M^{+}-M^{-}\right\|_{2}, \\
\|V\| \leqq 4 \sqrt{6} M_{0}^{1 / 2}\left\|M^{+}-M^{-}\right\|_{2}, \\
h_{n} \leqq 3 \pi \sqrt{L_{n}\left|M_{n}^{ \pm}\right| / 2} \leqq 6 \pi^{2} n\left(M_{n}^{+}-M_{n}^{-}\right), \text {for all } n \geqq 1, \\
L_{n} \leqq(4 \pi n)^{2}\left(M_{n}^{+}-M_{n}^{-}\right), \text {for all } n \geqq 1,
\end{gathered}
$$

hold. Suppose that $G_{m}$ is the first non-degenerate gap for some $m \geqq 1$. Then $M_{0} \geqq-M_{m}^{-}$.

The estimates (2.1-9) are new. It is interesting that some of them are linear. In the paper [KK2] there are the inverse estimates. For example,

$$
\left\|M^{ \pm}\right\|_{2} \leqq 4\left\|V_{1}\right\|\left(1+\left\|V_{1}\right\|\right) e^{3\left\|V_{1}\right\|} .
$$

Then we are able to estimate $\left\|V_{1}\right\|$ in terms of $\left\|M^{ \pm}\right\|_{2}$ and the inverse. Let us note that in the proof of [KK2] the Bernstein inequality was used. Hence the last estimate is "exponentially overstated." Unfortunately it is not clear how to get a more exact estimate (without exponent).

Let us consider the more general case. At first we give some definitions and facts from the conformal mapping theory. We call the set $K_{+}=\mathbf{C}_{+} \backslash \bigcup \gamma_{n}$ the comb where $\gamma_{n}=\left(p_{n}+i h_{n}, p_{n}-i h_{n}\right)$ is an excised slit with the height $h_{n} \geqq 0, n \in \mathbf{Z}, h_{0}=0$. Here $p_{n}$ is a strongly increasing sequence of real numbers such that $p_{n} \rightarrow \pm \infty$ as $n \rightarrow \pm \infty$. We call a conformal mapping $k(w)$ from the upper halfplane $\mathbf{C}_{+}$onto some comb $K_{+}$a general quasimomentum (GQ) if $k(0)=0$ and $k(i v)=i v(1+$ $o(1))$ as $v \rightarrow \infty$. It is well known that GQ $k$ is a continuous function of $w \in \overline{\mathbf{C}}_{+}$ [L]. In this case we introduce the sets

$$
\sigma_{n}=\left[a_{n-1}^{+}, a_{n}^{-}\right]=k^{-1}\left(\left[p_{n-1}, p_{n}\right]\right), \quad n \in \mathbf{Z} .
$$

We call the variable $w$ the momentum and $\sigma=\bigcup \sigma_{n}$ the spectrum of the momentum. We also denote by $g_{n}=\left(a_{n}^{-}, a_{n}^{+}\right)$a gap in the spectrum of the momentum and let $g=\bigcup g_{n}$. It is well known that the set $\sigma$ can not be the spectrum of two different momentum (see [L]). Let us note that the function $k(w)$ has the analytical continuation onto the domain $W=\mathbf{C} \backslash \bar{g}$ by the formula $k(\bar{w})=\bar{k}(w), w \in W$. We denote 
the length of the gap $g_{n}$ by $l_{n}$. We call GQ symmetric if $k(-w)=-k(w), w \in W$. For a symmetric GQ (SGQ) $k$ we define the energy $E=E(k)=w(k)^{2}$, where $w(k)$ is the inverse function for $k(w)$. If $k$ runs from $p_{n-1}$ to $p_{n}, n \geqq 1$, then $E(k)$ runs the segment $\Sigma_{n}=\left[A_{n-1}^{+}, A_{n}^{-}\right]$, where $A_{n}^{ \pm}=\left(a_{n}^{ \pm}\right)^{2}, n \geqq 1$. These segments are separated by the gap $G_{n}=\left(A_{n}^{-}, A_{n}^{+}\right), n \geqq 1$, and let $G=\bigcup G_{n}, K=\mathbf{C} \backslash \bigcup \gamma_{n}$. The function $E(k)$ is the conformal mapping from $K \cap\{\operatorname{Re} k>0\}$ onto the energy domain $\mathbf{E}=$ $C \backslash G$. With an edge of the gap $G_{n}$ having the length $L_{n}$ we associate the effective masses

$$
M_{n}^{ \pm}= \begin{cases}0, & \text { if } L_{n}=0, \\ 1 / E^{\prime \prime}\left(k\left(a_{n}^{ \pm}\right)\right), & \text {if } L_{n} \neq 0,\end{cases}
$$

and for the beginning of the energy spectrum we define the effective mass $M_{0}=$ $1 / E^{\prime \prime}(0)$.

For SGQ $k$ we introduce "an effective energy" $r(E)=k(\sqrt{E})^{2}, E \in \mathbf{C}_{+}$. The function $r(E)$ has the analytical continuation on the domain $\mathbf{E}$. The function $r$ is the conformal mapping from $\mathbf{E}$ onto the domain $\mathscr{R}=\mathbf{C} \backslash \Gamma$, where $\Gamma=\bigcup \Gamma_{n}$ and a parabolic slit $\Gamma_{n}=\left\{r=\left(p_{n}+i q\right)^{2},-h_{n}<q<h_{n}\right\}, n \geqq 1$. Let $k=p+i q, r=t+$ is and remark that $s(E)>0, E \in \mathbf{C}_{+}$. We introduce the moments

$$
Q_{n}=\frac{1}{\pi} \int_{g} w^{n} q(w) d w, \quad S_{n}=\frac{1}{\pi} \int_{G} E^{n} s(E) d E, \quad n \geqq-2,
$$

the real Hilbert spaces

$$
\ell_{m}^{2}=\left\{f=\left\{f_{n}\right\}_{1}^{\infty},\|f\|_{m}<\infty\right\}, \quad\|f\|_{m}^{2}=\sum_{n>0}\left(2 p_{n}\right)^{2 m} f_{n}^{2}, \quad m \geqq 0, \ell_{0}^{2}=\ell^{2},
$$

the integrals

$$
P_{2}^{+}=\frac{1}{\pi} \int p(t)^{2} q(t) d t, \quad P_{2}^{-}=\frac{1}{3 \pi} \int q(t)^{3} d t,
$$

and the Dirichlet integral for the function $f$ and the domain $\mathbf{C}$

$$
I_{D}(f)=\frac{1}{\pi} \iint\left|f^{\prime}(w)\right|^{2} d u d v, \quad w=u+i v .
$$

Here and below an integral with no limits indicated denotes the integration over $\mathbf{R}^{\mathbf{d}}, d \geqq 1$. Second, when we consider the functions $q(w), s(E)$ on the real line we take the functions $q(w)=q(w+i 0), s(E)=s(E+i 0)$. We have the following theorem about identities.

Theorem 2.3. Let $V \in L^{1}(0,1)$. Then the following identities:

$$
\begin{gathered}
I_{D}\left(\frac{k}{w}\right)=S_{-2}=2 M_{0}-1=2 Q_{-2}+Q_{-2}^{2}=-2 \sum_{n>0}\left(M_{n}^{+}+M_{n}^{-}\right), \\
I_{D}(k-w)=S_{-1}=2 Q_{0}=\int_{0}^{1} V(x) d x=2 \sum_{n>0}\left(A_{n}^{+} M_{n}^{+}+A_{n}^{-} M_{n}^{-}\right)
\end{gathered}
$$


hold. Let $V \in L^{2}(0,1)$. Then the following identities:

$$
\begin{aligned}
\frac{1}{4} I_{D}\left(k^{2}-E(k)\right) & =S_{0}=2 Q_{2}-Q_{0}^{2}=\int_{0}^{1} V_{1}(x)^{2} \frac{d x}{4} \\
& =-\frac{4}{3} Q_{0}^{2}+\frac{2}{3} \sum_{n>0}\left(\left(A_{n}^{+}\right)^{2} M_{n}^{+}+\left(A_{n}^{-}\right)^{2} M_{n}^{-}\right)
\end{aligned}
$$

hold. All series converge absolutely.

Let us show the relation SGQ with the differential operator (see [PT]). We consider the Schrödinger operator $T_{B}=-d^{2} / d x^{2}+V(x)$ in $L^{2}(\mathbf{R})$, where $V$ is a real potential from the Besikovitch space $B_{2}(\mathbf{R})$. Suppose that the spectrum of $T_{B}$ is absolute continuous and consists of the intervals $\Sigma_{n}=\left[A_{n-1}^{+}, A_{n}^{-}\right]$, where $A_{n-1}^{+}<$ $A_{n}^{-} \leqq A_{n}^{+}, n \geqq 1$, and let $A_{0}^{+}=0$. In the spectrum there are the gaps $G_{n}=\left(A_{n}^{-}, A_{n}^{+}\right)$ with the length $L_{n}=\left|G_{n}\right|$. We introduce the domain $W=\mathbf{C} \backslash \bigcup g_{n}$, where the interval $g_{n}=\left(a_{n}^{-}, a_{n}^{+}\right)=-g_{-n}, n \geqq 1$, and $a_{n}^{ \pm}=\sqrt{A_{n}^{ \pm}}>0$. Note that SGQ $k$ is defined uniquely as a conformal mapping from $W$ onto some domain (a comb) $K$ if $k(0)=0$ and $k(i v)=i v(1+o(1))$ as $v \rightarrow \infty$. Let $(\cdot, \cdot)_{B}$ be the scalar product in $B_{2}(\mathbf{R})$. Then (see [S])

$$
\begin{gathered}
2 Q_{0}=V_{0}=(V, 1)_{B}=\lim \int_{-a}^{a} V(x) \frac{d x}{2 a}, \quad a \rightarrow \infty, \\
8 Q_{2}=\|V\|_{B}^{2}=(V, V)_{B}=\lim \int_{-a}^{a} V(x)^{2} \frac{d x}{2 a}, \quad a \rightarrow \infty .
\end{gathered}
$$

Let $V_{1}=V-V_{0}$, then $\left\|V_{1}\right\|_{B}^{2}=8 Q_{2}-4 Q_{0}^{2}$. For GQ we define the function $w_{1}=$ $k w(k)-E(k)$ and let $h_{+}=\sup h_{n}$. We have the following theorem on the identities.

Theorem 2.4. Let $k$ be SGQ. Then

$$
I_{D}\left(\frac{k}{w}\right)=S_{-2}=2 M_{0}-1=2 Q_{-2}+Q_{-2}^{2} .
$$

Let $Q_{0}<\infty$. Then

$$
I_{D}(k-w)=S_{-1}=2 Q_{0}
$$

Let $Q_{2}<\infty$. Then

$$
\begin{gathered}
I_{D}\left(E-k^{2}\right)=S_{0}, \\
2 Q_{2}=S_{0}+Q_{0}^{2}=I_{D}\left(w_{1}\right)-Q_{0}^{2}=\frac{1}{2}\left(I_{D}\left(w_{1}\right)+S_{0}\right)=2\left(P_{2}^{+}+Q_{0}^{2}-P_{2}^{-}\right) \\
P_{2}^{-}=P_{2}^{+}+Q_{2}-S_{0}=\frac{1}{\pi} \int(p(t)-t)^{2} q(t) d t \\
\frac{Q_{0}^{3}}{4 Q_{2}} \leqq h_{+}^{2} \leqq 2 Q_{0}=S_{-1} \leqq 2 \sqrt{2 Q_{2}} .
\end{gathered}
$$

Now we consider the estimates for GQ. We have the theorem. 
Theorem 2.5. Let $k$ be SGQ and $Q_{2}<\infty$. Then

$$
\begin{gathered}
\|L\| \leqq \min \left\{\frac{16}{\pi} M_{0}\|h\|_{1}, 2^{7} 9 M_{0}^{2}\|M\|_{2}, 4\left\|M^{+}-M^{-}\right\|_{2}\right\}, \\
2\|h\|_{1}^{2} \leqq 9 \pi^{2}\|L\|\|M\|_{2}, \\
S_{0} \leqq \frac{1}{\pi}\|h\|_{1}\|L\| \leqq \min \left\{4^{5} 9^{2} M_{0}^{3}\|M\|_{2}^{2}, 12 \sqrt{2}\left\|M^{+}-M^{-}\right\|_{2}^{2}\right\}, \\
Q_{2} \leqq M_{0} S_{0} .
\end{gathered}
$$

Let us present the following "local estimates."

Theorem 2.6. i) Let $k$ be SGQ and a cut $(p-i h, p+i h), p>0$, correspond to the energy gap $G=\left(A^{-}, A^{+}\right), L=|G|$, with the effective masses $M^{ \pm}$. Then

$$
\begin{gathered}
q(\sqrt{E})^{2} \leqq 2 L\left|M^{ \pm}\right| \min \left\{\frac{9 \pi^{2}}{4}, \frac{\left|E-A^{ \pm}\right|}{\left|E-A^{\mp}\right|}\right\}, \quad E \in G, \\
2 h^{2} \leqq 9 \pi^{2} L\left|M^{ \pm}\right|, \\
\frac{h^{2}}{2\left|M^{-} M^{+}\right|^{1 / 2}} \leqq L \leqq 8 p^{2}\left(\sqrt{M^{+}}+\sqrt{-M^{-}}\right)^{2} .
\end{gathered}
$$

ii) Let $k^{(1)}, k^{(2)}$, be two SGQ and $\gamma_{n}^{(1)} \subset \gamma_{n}^{(2)}$ for all $n \geqq 1$. Then $\left|\Sigma_{n}^{(1)}\right|>$ $\left|\Sigma_{n}^{(2)}\right|,\left|M_{n}^{(1) \pm}\right|<\left|M_{n}^{(2) \pm}\right|$ for all $n \geqq 1$, and $Q_{0}^{(1)}<Q_{0}^{(2)}, S_{0}^{(1)}<S_{0}^{(2)}$.

iii) Suppose that $\Sigma_{n}^{(2)} \subset \Sigma_{n}^{(1)}$ for all $n>0$, and $A_{m}^{(1)+}=A_{m}^{(2)+}$ for some $m>0$ (or $A_{m}^{(1)-}=A_{m}^{(2)-}$ ). Then $M_{m}^{(1)+} \leqq M_{m}^{(2)+}\left(\right.$ or $\left.\left|M_{m}^{(1)-}\right| \leqq\left|M_{m}^{(2)-}\right|\right)$.

iv) Let $\left(0, A_{1}^{-}\right)$be the first energy spectral band and $M_{0}, M_{1}^{-}$be the corresponding effective masses. Then $M_{0} \geqq-M_{1}^{-}$.

By ii) of this theorem we see that if some height $h_{n}$ increases then all spectral bands decrease, all effective masses and two moments increase.

\section{The Local Properties of the Effective Energy}

We shall consider the symmetric GQ, i.e. $k(-w)=-k(w), w \in W$. Let $E=\xi+i \eta$, $k=p+i q, w=u+i v, E=w^{2}, r=r(E)=k(\sqrt{E})^{2}=t+i s$. We have

$$
\begin{aligned}
r= & (p+i q)^{2}=t+i s \in \mathbf{C}_{+}, \quad t=p^{2}-q^{2}, \quad s=2 p q \geqq 0, \\
& \text { if } k=p+i q, \quad q>0, p>0 .
\end{aligned}
$$

We have $s(E)=\eta(1+o(1))$ as $\eta \rightarrow \infty$. Hence by the Herglotz theorem we obtain

$$
s(E)=\eta\left(1+\frac{1}{\pi} \int_{0}^{\infty} \frac{s(y) d y}{|y-E|^{2}}\right), \quad E \in \mathbf{C}_{+} .
$$


By (3.1) we get

$$
s_{\eta}^{\prime}(\xi, 0)=1+\frac{1}{\pi} \int_{0}^{\infty} \frac{s(y) d y}{(y-E)^{2}}=r^{\prime}(E)>1, \quad E=\xi \in \Sigma=\mathbf{R} \backslash G .
$$

Hence

$$
\begin{gathered}
r^{(m)}(\xi)=\frac{m !}{\pi} \int_{0}^{\infty} \frac{s(y) d y}{(y-E)^{m+1}}, \quad \xi \in \Sigma, m \geqq 2, \\
r^{(2 m+1)}(\xi)>0, \quad \xi \in \Sigma, m \geqq 0,
\end{gathered}
$$

and the function $r^{\prime}(\xi), \xi \in \Sigma_{n}$, is convex upward. We present the following result. Lemma 3.1. i) Let $k$ be SGQ and $E \in \mathrm{C}_{+}$. Then

$$
\begin{aligned}
r(E) & =E+C+\frac{1}{\pi} \int_{0}^{\infty} s(y)\left(\frac{1}{y-E}-\frac{y}{1+y^{2}}\right) d y, \\
C & =-\frac{1}{\pi} \int_{0}^{\infty} \frac{s(y) d y}{y\left(1+y^{2}\right)} .
\end{aligned}
$$

Let in addition $S_{-1}<\infty$. Then

$$
r(E)=E-S_{-1}+\frac{1}{\pi} \int_{0}^{\infty} \frac{s(y) d y}{(y-E)}, \quad E \in \mathbf{E} .
$$

ii) Let $k$ be SGQ and a cut $(p+i h, p-i h)$ correspond to an energy gap $G=\left(A^{-}, A^{+}\right), L=|G|$, and $M^{ \pm}$be the corresponding effective masses. Then

$$
\begin{gathered}
\frac{1}{\pi} \int_{G}\left(\frac{1}{y-A^{-}}+\frac{1}{A^{+}-y}\right) s(y) d y=L\left(1+\frac{1}{\pi} \int_{\mathbf{R}_{+} \backslash G} \frac{s(y) d y}{\left(y-A^{-}\right)\left(y-A^{+}\right)}\right), \\
q(\sqrt{E})^{2}<2 L\left|M^{ \pm}\right| \frac{\left|E-A^{ \pm}\right|}{\left|E-A^{\mp}\right|}, \quad A^{-}<E<A^{+}, \\
\frac{1}{3} h L<\int_{A^{-}}^{A^{+}} q(\sqrt{E}) d E \leqq L \min \left\{h, \pi \sqrt{\frac{L\left|M^{ \pm}\right|}{2}}\right\} .
\end{gathered}
$$

Proof. i) The similar formula of (3.5) for $k(w)$ is proved in [MO]. The proof for $r(E)$ is the same. By (3.5) we get (3.7).

ii) By (3.5) we have

$$
r\left(A^{ \pm}\right)=A^{ \pm}+C+\frac{1}{\pi} \int_{0}^{\infty}\left(\frac{1}{y-A^{ \pm}}-\frac{y}{1+y^{2}}\right) s(y) d y,
$$

and by $r\left(A^{+}\right)=r\left(A^{-}\right)$we obtain (3.8).

In [KK1] there is the following estimate. Let a function $f$ be harmonic and positive in the domain $D=C_{+} \cup C_{-} \cup G$. Suppose that $f(E)^{2}=\left(A^{+}-E\right)(2 B+$ $o(1))$ as $E \uparrow A^{+}$. Then

$$
f(E)^{2}<2 L B \frac{A^{+}-E}{E-A^{-}}, \quad A^{-}<E<A^{+} .
$$


We take $f=q(\sqrt{E})$. It is clear that $q(\sqrt{E})>0, E \in D$. By the definition of the effective masses we have $q(\sqrt{E})^{2}=\left(A^{+}-E\right)\left(2 M^{+}+o(1)\right)$ as $E \uparrow A^{+}$. Hence we get (3.9). The proof for $M^{-}$is the same.

We integrate the estimate (3.9) on the interval $G$ and we have

$$
\int_{G} q(\sqrt{E}) d E \leqq \sqrt{2 L \mid M^{ \pm}} \int_{G} \sqrt{\frac{A^{+}-E}{E-A^{-}}} d E=\sqrt{2 L\left|M^{ \pm}\right|} \frac{\pi L}{2} .
$$

Moreover we have a simple estimate $\int_{G} q(\sqrt{E}) d E \leqq h L$ and then we get the second estimate in (3.10). We have to show the first one. The function $q(w), w \in$ $\left(\sqrt{A^{-}}, \sqrt{A^{+}}\right)$is convex (see [KK1]). Suppose that $q\left(a_{0}\right)=h$ for some $a_{0}^{2} \in G$, and let $a_{ \pm}^{2}=A^{ \pm}, q_{ \pm}=h /\left(a_{0}-a_{ \pm}\right)$. Let us define the function

$$
q_{1}= \begin{cases}q_{-}\left(w-a_{-}\right), & \text {if } a_{-}<w<a_{0}, \\ q_{+}\left(w-a_{+}\right), & \text {if } a_{0}<w<a_{+}\end{cases}
$$

Then $q(w) \geqq q_{1}(w), a_{-}<w<a_{+}$. Hence

$$
\begin{aligned}
\int_{G} q(\sqrt{E}) d E & \geqq \int_{a_{-}}^{a_{+}} q_{1}(w) d w^{2} \\
& =-\int_{a_{-}}^{a_{+}} q_{1}(w)^{\prime} w^{2} d w=-\int_{a_{-}}^{a_{0}} q_{-} w^{2} d w-\int_{a_{0}}^{a_{+}} q_{+} w^{2} d w \\
& =\frac{h}{3}\left(a_{+}^{2}-a_{-}^{2}+a_{0}\left(a_{+}-a_{-}\right)\right) \geqq \frac{1}{3} h L
\end{aligned}
$$

and we get (3.10).

By Lemma 3.1 we get the main local estimates for SGQ in the following

Corollary 3.2. Let $k$ be $\mathrm{GQ}$ and a cut $(p+i h, p-i h)$ correspond an energy gap $G=\left(A^{-}, A^{+}\right), L=|G|$, and $M^{ \pm}$be the corresponding effective masses. Then

$$
\begin{gathered}
q(\sqrt{E})^{2}<2 L\left|M^{-} M^{+}\right|^{1 / 2}, \quad A^{-}<E<A^{+}, \\
h^{2}<\frac{(3 \pi)^{2}}{2} L\left|M^{ \pm}\right|, \\
L<8 p^{2}\left(\sqrt{M^{+}}+\sqrt{-M^{-}}\right)^{2} \leqq 4(2 p)^{2}\left(M^{+}-M^{-}\right) .
\end{gathered}
$$

Proof. By (3.9) we get (3.11) and by (3.10) we obtain (3.12).

Let $P=2 p\left(\sqrt{2 L M^{+}}+\sqrt{-2 L M^{-}}\right)$. Then by (3.8), (3.9) we have

$$
L \leqq \frac{P}{\pi} \int_{G} \frac{d E}{\sqrt{\left(E-A^{-}\right)\left(A^{+}-E\right)}}=P,
$$

and hence we get (3.13). 
We prove the following main theorem.

Proof of Theorem 2.6. i) By (3.9),(3.12) we obtain (2.25), (2.26).

The estimate (2.27) follows from (3.11), (3.13).

ii) We introduce the function $f(E)=\operatorname{Im} E_{2}\left(r_{1}(E)\right), E \in \mathbf{C}_{+}$. This function is harmonic, non-negative in $\mathbf{C}_{+}$and continuous in $\overline{\mathbf{C}}_{+}$. Suppose the inequality

$$
f(E)=\operatorname{Im} E_{1}\left(r_{2}(E)\right) \geqq \operatorname{Im} E_{2}\left(r_{2}(E)\right)=\eta, \quad E=\xi+i \eta .
$$

Then we obtain $\operatorname{Im} E_{1} \geqq \operatorname{Im} E_{2}$ in the domain $r_{2}\left(\mathbf{C}_{+}\right)$. Moreover we have

$$
E_{1}(r)^{\prime}=\frac{\partial}{\partial s} \operatorname{Im} E_{1}(r) \geqq \frac{\partial}{\partial s} \operatorname{Im} E_{2}(r)=E_{2}(r)^{\prime}, \quad r \in \mathbf{R}, r \neq r_{n} \equiv p_{n}^{2} .
$$

Hence we obtain

$$
\left|\Sigma_{n}^{(1)}\right|=\int_{r_{n-1}}^{r_{n}} E_{1}^{\prime}(r) d r \geqq \int_{r_{n-1}}^{r_{n}} E_{2}^{\prime}(r) d r=\left|\Sigma_{n}^{(2)}\right|, \quad n \geqq 1 .
$$

Moreover we have for $\alpha=1,2$, that

$$
E_{\alpha}-A_{n}^{(\alpha)+}=\int_{r_{n}}^{r} E_{\alpha}^{\prime}(r) d r=\frac{\left(r-r_{n}\right)^{2}}{\left(2 p_{n}\right)^{2} 2 M_{n}^{(\alpha)+}}(1+o(1)), \quad \text { as }\left(r-r_{n}\right) \searrow 0,
$$

hence we get $\left|M_{m}^{(1)+}\right| \leqq\left|M_{m}^{(2)+}\right|$. The proof for $M_{n}^{(\alpha)-}$ is the same.

Let us prove (3.14). By the representation (2.15) we obtain that

$$
r_{m}(E)=E(1+o(1)), \quad E \in U(A)=\{E: \eta>A|\xi|\}, \quad|E| \rightarrow \infty .
$$

But for any $A$ there exists a constant $R=R(A)>0$ such that

$$
r_{m}(U(A)) \supset\{E:|E|>R\} \cap U(2 A), \quad m=1,2 .
$$

Hence $E_{\alpha}(r)=r(1+o(1)), r \in U(2 A),|r| \rightarrow \infty$, and

$$
\left.E_{1}\left(r_{2}(i \eta)\right) / i \eta=\left[E_{1}\left(r_{2}(i \eta)\right) /\left(r_{2}(i \eta)\right)\right]\left[r_{2}(i \eta)\right) / i \eta\right] \rightarrow 1, \quad \text { as } \eta \rightarrow \infty .
$$

From this it follows that $f(i \eta)=\eta(1+o(1))$, as $\eta \rightarrow \infty$, and by the Herglotz theorem we obtain (3.14).

Using (4.1-3) and the maximum principle we get the estimates for $Q_{0}, Q_{2}, S_{0}$.

iii) By the maximum principle we have the inequality $s_{1}(E) \leqq s_{2}(E), E \in \mathbf{R}$. Then by the definition of the effective mass we obtain

$$
\begin{aligned}
M_{m}^{(1)+} & =\lim \frac{s_{1}(E)^{2}}{2\left(2 p_{n}\right)^{2}\left(A_{m}^{(1)+}-E\right)} \\
& \leqq \lim \frac{s_{2}(E)^{2}}{2\left(2 p_{n}\right)^{2}\left(A_{m}^{(1)+}-E\right)}=M_{m}^{(2)+}, \quad \text { as } E \uparrow A_{m}^{(1)+} .
\end{aligned}
$$

iv) In $[\mathrm{K} 1]$ there is the estimate $k^{\prime}(0) \geqq \sqrt{-2 M_{1}^{-}}$and the equality $k^{\prime}(0)^{2}=$ $2 M_{0}$. Then we get $M_{0} \geqq-M_{1}^{-}$. 


\section{The Identities}

In this section we find some identities which are basic for us. First we present the identity about $S_{-1}, S_{0}, Q_{2}, Q_{0}, \ldots$

Lemma 4.1. Let $k$ be SGQ and $Q_{2 m}<\infty$, for some $m \geqq 0$. Then

$$
r(E)=E-S_{-1}-\frac{S_{0}}{E}-\frac{S_{1}}{E^{2}}-\cdots-\frac{S_{m-1}+o(1)}{E^{m}}, \quad \text { as }|E| \rightarrow \infty,|\eta|>\delta \xi,
$$

for some $\delta>0$ and here

$$
\begin{gathered}
S_{-1}=2 Q_{0}, \quad S_{0}=2 Q_{2}-Q_{0}^{2}, \\
S_{1}=2 Q_{4}-2 Q_{0} Q_{2}, \ldots, S_{m-1}=2 Q_{2 m}-\sum_{0}^{m-1} Q_{2 m-2-2 p} Q_{2 p} .
\end{gathered}
$$

Proof. We have the asymptotics (see [KK1])

$$
k(w)=w-\frac{Q_{0}}{w}-\frac{Q_{2}}{w^{3}}-\cdots-\frac{Q_{2 m}+o(1)}{w^{2 m+1}}, \quad \text { as }|w| \rightarrow \infty, w=u+i v,|u|>\varepsilon v,
$$

for some $\varepsilon>0$. Hence we get

$$
\begin{aligned}
r-E=(k+w)(k-w)= & -\left(2-Q_{0} E^{-1}-Q_{2} E^{-2}-\cdots-Q_{2 m} E^{-m-1}-\cdots\right) \\
& \times\left(Q_{0}+Q_{2} E^{-1}+\cdots+Q_{2 m} E^{-m}+\cdots\right) \\
= & -2 Q_{0}-\left(2 Q_{2}-Q_{0}^{2}\right) E^{-1}-2\left(Q_{4}-Q_{0} Q_{2}\right) E^{-2}-\cdots \\
& -\left(2 Q_{2 m}-\sum_{0}^{m-1} Q_{2 m-2-2 p} Q_{2 p}+o(1)\right) E^{-m}
\end{aligned}
$$

By (3.7) we get

$$
\begin{aligned}
r(E)-E+S_{-1} & =-\frac{1}{\pi E} \int \frac{s(y) d y}{1-(y / E)} \\
& =-\frac{S_{0}}{E}-\frac{S_{1}}{E^{2}}-\cdots-\frac{S_{m-1}+o(1)}{E^{m}}, \quad \text { as }|E| \rightarrow \infty .
\end{aligned}
$$

If we compare these two asymptotics we get (4.2).

Later on in this section we need the following result.

Lemma 4.2. Let $k$ be SGQ and finite gap. Then for any $m \geqq 0$ the following asymptotics hold:

$$
w=k+\frac{P_{0}}{k^{1}}+\frac{P_{2}}{k^{3}}+\frac{P_{4}}{k^{5}}+\cdots \frac{P_{2 m}+o(1)}{k^{2 m+1}}, \quad \text { as }|k| \rightarrow \infty,
$$

and here

$$
P_{0}=Q_{0}, \quad P_{2}=P_{2}^{+}-P_{2}^{-}=Q_{2}-Q_{0}^{2}, \ldots
$$


Proof. We have the finite number of gaps. Then $g=\bigcup g_{n} \subset[-a, a]$ for some large $a$. We take the function $f(k)=w(k)-k$ and we get $f(k)=o\left(k^{-1}\right)$, as $|k| \rightarrow \infty$. The function $f$ is analytic in the domain $|k|>a$. Then for $|k|>a$ we obtain

$$
\begin{aligned}
f(k) & =-\frac{1}{k 2 \pi i} \int_{|k|=a} \frac{f(t) d t}{1-t / k} \\
& =-\frac{1}{k 2 \pi i} \sum_{n \geqq 0}^{2 m} \int_{|k|=a} f(t) t^{n} d t k^{-1-n}+\cdots=\frac{P_{0}}{k}+\frac{P_{1}}{k^{2}}+\frac{P_{2}}{k^{3}}+\cdots+,
\end{aligned}
$$

where

$$
P_{n}=-\frac{1}{2 \pi i} \int_{|k|=a} f(t) t^{n} d t
$$

Integrating by paths we obtain

$$
P_{n}=\frac{1}{2 \pi i(n+1)} \int_{|k|=a} k^{n+1}(w) d w .
$$

Then

$$
P_{0}=\frac{1}{2 \pi i} \int_{|k|=a} k(w) d w=\frac{1}{2 \pi i} \int_{g}(k(w)-\bar{k}(w)) d w=\frac{1}{\pi} \int_{g} q(w) d w=Q_{0},
$$

and

$$
P_{1}=\frac{1}{2 \pi i 2} \int_{|k|=a} k(w)^{2} d w=\frac{1}{4 \pi i} \int_{g}\left(k(w)^{2}-\bar{k}^{2}(w)\right) d w=\frac{1}{\pi} \int_{g} p(w) q(w) d w .
$$

Moreover by $k^{3}=(p+i q)^{3}=\left(p^{3}-3 p q^{2}\right)+i\left(3 p^{2} q-q^{3}\right)$, we get

$$
\begin{aligned}
P_{2} & =\frac{1}{6 \pi i} \int_{|k|=a} k(w)^{3} d w=\frac{1}{6 \pi i} \int_{g}\left(k(w)^{3}-\bar{k}^{3}(w)\right) d w \\
& =\frac{1}{3 \pi} \int_{g}\left(3 p^{2} q-q^{3}\right) d w=P_{2}^{+}-P_{2}^{-},
\end{aligned}
$$

and so on. We get (4.3).

Now we find the asymptotics $w(k)$ as $|k| \rightarrow \infty$ in terms of $Q_{n}, n>-1$. Indeed we have got the asymptotics in terms of $P_{n}, n \geqq 0$,

$$
w=k+P_{0} k^{-1}+P_{2} k^{-3}+P_{4} k^{-5}+\cdots+\left(P_{2 m}+o(1)\right) k^{-2 m-1}, \quad \text { as }|k| \rightarrow \infty,
$$

and there is the asymptotics in [KK1]

$$
k=w-\frac{Q_{0}}{w}-\frac{Q_{2}}{w^{3}}-\frac{Q_{4}}{w^{5}}-\cdots-\frac{Q_{2 m}+o(1)}{w^{2 m+1}}, \quad \text { as }|w| \rightarrow \infty,
$$

If we compare two asymptotics we get (4.4). 
Remark that it is possible to get the asymptotics (4.3) for the more general case than a finite gap case. But in the present paper it is enough to obtain the needed identities (4.4).

We shall prove Theorem 2.4 in Lemma 4.3-5.

Lemma 4.3. Suppose that $k$ be SGQ. Then we have (2.15), i.e.

$$
2 M_{0}-1=S_{-2}=\left(2+Q_{-2}\right) Q_{-2}=I_{D}\left(\frac{k}{w(k)}\right) .
$$

Let in addition $Q_{0}<\infty$. Then we have (2.16), i.e.

$$
I_{D}(k-w)=2 Q_{0}=S_{-1} .
$$

Moreover

$$
S_{-1}^{2}=4 Q_{0}^{2} \leqq S_{-2} S_{0}=\left(2 M_{0}-1\right) S_{0} .
$$

Proof. By (3.2) and by $r^{\prime}(0)=2 M_{0}$ at $E=0$ we get

$$
2 M_{0}=1+\frac{1}{\pi} \int_{0}^{\infty} \frac{s(y) d y}{y^{2}}=1+S_{-2} .
$$

In [K1] we get $2 M_{0}=\left(1+Q_{-2}\right)^{2}$. Then we have the next identity in (4.7). It is well known (see [Koo]) that $I_{D}\left(w_{-1}\right)=S_{-2}$, and hence we get (4.7). In [KK1] there is the equality $I_{D}(k-w)=2 Q_{0}$. By (4.2) we have $2 Q_{0}=S_{-1}$ and by the Cauchy inequality we obtain $S_{-1}^{2} \leqq S_{0} S_{-2}$ and by (4.7) we get (4.9).

We shall prove the identities for the case $Q_{2}<\infty$ in Theorem 2.4.

Lemma 4.4. Suppose that $k$ be SGQ and $Q_{2}<\infty$. Then we have (2.17-19), i.e.

$$
\begin{gathered}
P_{2}^{+}-P_{2}^{-}=Q_{2}-Q_{0}^{2} \\
P_{2}^{-}=P_{2}^{+}+Q_{2}-S_{0}=\frac{1}{\pi} \int(p(t)-t)^{2} q(t) d t \\
\pi S_{0}=\iint_{\mathbf{C}_{+}}\left|r(E)^{\prime}-1\right|^{2} d \xi d \eta=\frac{1}{2} \iint_{\operatorname{Re} k>0}\left|\left(k^{2}-w(k)^{2}\right)^{\prime}\right|^{2} d p d q=\int_{0}^{\infty} s(E) d E \\
2 Q_{2}=S_{0}+Q_{0}^{2}=\frac{1}{2}\left(I_{D}\left(w_{1}\right)+S_{0}\right)=I_{D}\left(w_{1}\right)-Q_{0}^{2}
\end{gathered}
$$

Proof. We proved (4.10) for the finite gap GQ in (4.4). Let us consider the general case. Introduce the set $\sigma^{N}=\sigma \cup(-\infty,-N) \cup(N, \infty)$ and the variable corresponding to $\sigma^{N}$ we denote by the upper index $N$. It is well known (see [L]) that $q^{N}(u) \nearrow q(u),\left|p^{N}(u)\right| \nearrow|p(u)|$, as $N \rightarrow \infty, u \in \mathbf{R}$. Then by Levy's Theorem we obtain $Q_{0}^{N} \nearrow Q_{0}, Q_{2}^{N} \nearrow Q_{2}, P_{2}^{ \pm, N} \nearrow P_{2}^{ \pm}$, as $N \rightarrow \infty$. Then we get (4.10) for GQ.

By (4.2) and (4.10) we have (4.11).

By the Green formula we have

$$
\iint_{\mathbf{C}_{+}}\left|r(E)^{\prime}-1\right|^{2} d \xi d \eta=-\int_{-\infty}^{\infty}(s-\eta)(t-\xi)_{\xi}^{\prime} d \xi=\int_{0}^{\infty} s\left(1-t_{\xi}^{\prime}\right) d \xi=\int_{0}^{\infty} s(\xi) d \xi,
$$

and by symmetry we obtain (4.12).

We have the first equality in (4.2). In [KK1] there is the formula $I_{D}\left(w_{1}\right)+S_{0}=$ $4 Q_{2}$ and by (4.2) we obtain (4.13). 
Now we obtain the estimates for general quasimomentum.

Lemma 4.5. i) Let $k$ be GQ and $Q_{0}<\infty$. Then $h_{+}^{2} \leqq 2 Q_{0}$. ii) Let in addition $k$ be SGQ and $Q_{2}<\infty$. Then

$$
\begin{gathered}
h_{+}^{4} \leqq 4 Q_{0}^{2} \leqq 8 Q_{2}, \\
\frac{Q_{0}^{4}}{4 Q_{2}} \leqq P_{2}^{-} \leqq \frac{1}{3} h_{+}^{2} Q_{0} \leqq \frac{2}{3} Q_{0}^{2}, \\
\frac{3 Q_{0}^{3}}{4 Q_{2}} \leqq h_{+}^{2} \leqq 2 Q_{0}=S_{-1} \leqq 2 \sqrt{2 Q_{2}}, \\
P_{2}^{+}+\frac{1}{3} Q_{0}^{2} \leqq Q_{2} \leqq P_{2}^{+}+Q_{0}^{2}, \\
\frac{1}{16}\|l\|_{1}^{2} \leqq P_{2}^{+} \leqq \frac{1}{2} S_{0}+\frac{1}{18} h_{+}^{4}, \\
\frac{1}{\pi} \sum_{n \geqq 1} p_{n}^{2} h_{n} l_{n} \leqq P_{2}^{+} \leqq \frac{2}{\pi} \sum_{n \geqq 1} p_{n}^{2} h_{n} l_{n} .
\end{gathered}
$$

Proof. Let $Q_{0}<\infty$. First we consider the finite gap case. Suppose that $h_{+}=h_{m}$ for some $m \in \mathbf{Z}$. We have a simple domain $K^{(m)}=\mathbf{C} \backslash \gamma_{m}$. It is well known that in the finite gap case for the corresponding moments there is an inequality $Q_{0}^{(m)} \leqq Q_{0}$ (see [J]). But we have exactly $Q_{0}^{(m)}=h_{m}^{2} / 2$. Then we get $h_{+}^{2}=h_{m}^{2} \leqq 2 Q_{0}$.

Let us consider the general case.

Suppose that $h_{+}=h_{m}$ for some $m \in \mathbf{Z}$.

Introduce the set $\sigma^{(N)}=\sigma \cup(-\infty,-N) \cup(N, \infty)$ and the variable corresponding to $\sigma^{(N)}$ we denote by the upper index $N$. Then by the finite gap case we get $\left(h_{+}^{(N)}\right)^{2} \leqq 2 Q_{0}^{(N)}$. It is well known (see [L]) that $q^{(N)}(u) / q(u)$, as $N \rightarrow \infty, u \in \mathbf{R}$. Then $h_{+}^{(N)} \rightarrow h_{+}$and by Levy's Theorem we obtain $Q_{0}^{(N)} / Q_{0}$, as $N \rightarrow \infty$. Then $h_{+}^{2}=\lim \left(h_{+}^{(N)}\right)^{2} \leqq \lim 2 Q_{0}^{(N)}=2 Q_{0}$, as $N \rightarrow \infty$. Hence we get $h_{+}^{2} \leqq 2 Q_{0}$.

Let $Q_{2}<\infty$. Then by (4.2) we have (4.14).

By (4.2), (4.11) we get

$$
Q_{0}^{2}=2 Q_{2}-S_{0}=\frac{2}{\pi} \int(t-p) t q d t \leqq 2 \sqrt{P_{2}^{-} Q_{2}}
$$

Then we get $Q_{0}^{4} \leqq 4 P_{2}^{-} Q_{2}$. By (4.14) we have

$$
P_{2}^{-}=\frac{1}{3 \pi} \int q(t)^{3} d t \leqq \frac{h_{+}^{2}}{3 \pi} \int q(t) d t=\frac{h_{+}^{2} Q_{0}}{3} \leqq \frac{2}{3} Q_{0}^{2},
$$

and we get (4.15).

By (4.15) we have $3 Q_{0}^{4} / Q_{2} \leqq 4 h_{+}^{2} Q_{0}$ and by (4.14) we get the second estimate in (4.16). By (4.2) we have $2 Q_{0}=S_{-1}$ and by (4.14) we get the last estimate in (4.16).

By (4.10), (4.15) we have $P_{2}^{+}+Q_{0}^{2}=Q_{2}+P_{2}^{-} \leqq Q_{2}+(2 / 3) Q_{0}^{2}$ and we get the first estimate in (4.17) and by (4.10) we obtain the second one. 
By the definition of $P_{2}^{+}$we have

$$
P_{2}^{+}=\frac{1}{\pi} \int p(t)^{2} q(t) d t=\frac{1}{\pi} \sum \int_{g_{n}} p_{n}^{2} q(t) d t
$$

and by $8 \int_{g_{n}} q(t) d t \geqq \pi l_{n}^{2}$ (see $\left.[\mathrm{KK} 1]\right)$ we get $16 P_{2}^{+} \geqq\|l\|_{1}^{2}$.

By (4.11), (4.15) we have

$$
2 P_{2}^{+}+Q_{0}^{2}=S_{0}+2 P_{2}^{-} \leqq S_{0}+\frac{2}{3} h_{+}^{2} Q_{0} \leqq S_{0}+Q_{0}^{2}+\frac{1}{9} h_{+}^{4},
$$

then we have $2 P_{2}^{+} \leqq S_{0}+\frac{1}{9} h_{+}^{4}$.

By (4.20) and by $l_{n} h_{n} / 2 \leqq \int_{g_{n}} q(t) d t \leqq l_{n} h_{n}$ (see [KK1]) we obtain (4.19).

\section{The Estimates for SGQ and the Hill Operator}

We prove the estimates for GQ. Let us introduce the scalar product $(\cdot, \cdot)$ in $\ell^{2}$ and a number $H_{n}=2 p_{n} h_{n}, n \geqq 1$, and the sequence $H=\left\{H_{n}, n \geqq 1\right\}$.

Theorem 5.1. Let $k$ be SGQ and $Q_{2}<\infty$. Then

$$
\begin{gathered}
\frac{1}{16}\|L\|^{2} \leqq Q_{2} \leqq M_{0} S_{0}, \\
\frac{(H, L)}{3 \pi} \leqq S_{0} \leqq \frac{(H, L)}{\pi} \leqq \frac{\|H\|\|L\|}{\pi}, \\
S_{0} \leqq \frac{16}{\pi^{2}} M_{0}\|H\|^{2} \leqq(4 \cdot 72)^{2} M_{0}^{3}\|M\|_{2}^{2}, \\
\|L\| \leqq \frac{16}{\pi} M_{0}\|H\| \leqq 2(24)^{2} M_{0}^{2}\|M\|_{2}, \\
\|H\| \leqq \frac{3 \pi}{\sqrt{2}}\|L\|^{1 / 2}\|M\|_{2}^{1 / 2} \leqq 72 \pi M_{0}\|M\|_{2} .
\end{gathered}
$$

Proof. In [KK1] there is the first estimate of (5.1). By (4.2), (4.9) we have $2 Q_{2}+$ $3 Q_{0}^{2}=S_{0}+4 Q_{0}^{2} \leqq S_{0}+\left(2 M_{0}-1\right) S_{0}=2 M_{0} S_{0}$, and then we get $(5.1)$.

We have

$$
S_{0}=\frac{2}{\pi} \int_{0}^{\infty} p q d E=\sum_{n \geqq 1} \frac{2}{\pi} \int_{G_{n}} p_{n} q d E .
$$

By (3.10) we get $h_{n} L_{n} / 3 \leqq \int_{G_{n}} q d E \leqq h_{n} L_{n}$ and we obtain (5.2).

By (5.1) we have $\|L\|^{2} \leqq 16 Q_{2} \leqq 16 M_{0} S_{0} \leqq 16 M_{0}\|H\|\|L\| / \pi$. Then we obtain $\pi\|L\| \leqq 16 M_{0}\|H\|$ and by (3.12) we have $H_{n}^{2} \leqq 2(3 \pi / 2)^{2}\left(2 p_{n}\right)^{2} M_{n} L_{n}$. Hence we get (5.3). Moreover we have the first estimate in (5.4) and by (5.3) we have (5.4).

By (5.2-4) we obtain (5.5).

We prove "linear estimates". 
Theorem 5.2. Let $k$ be $\mathrm{SGQ}$ and $Q_{2}<\infty$. Then

$$
\begin{gathered}
\|L\| \leqq 4\left\|M^{+}-M^{-}\right\|_{2}, \\
\|H\|^{2} \leqq 18 \pi^{2}\|M\|_{2}\left\|M^{+}-M^{-}\right\|_{2}, \\
S_{0} \leqq 12 \sqrt{2}\|M\|_{2}^{1 / 2}\left\|M^{+}-M^{-}\right\|_{2}^{3 / 2} \leqq 12\left\|M^{+}-M^{-}\right\|_{2}^{2}, \\
Q_{2} \leqq 12 \sqrt{2} M_{0}\|M\|_{2}^{1 / 2}\left\|M^{+}-M^{-}\right\|_{2}^{3 / 2} \leqq 12 M_{0}\left\|M^{+}-M^{-}\right\|_{2}^{2} .
\end{gathered}
$$

Proof. By (3.13) we have $L_{n} \leqq 4\left(2 p_{n}\right)^{2}\left(M_{n}^{+}-M_{n}^{-}\right)$. Then we get (5.6).

By (5.4), (5.6) we get $2\|H\|^{2} \leqq 9 \pi^{2}\|L\|\|M\|_{2} \leqq 36 \pi^{2}\|M\|_{2}\left\|M^{+}-M^{-}\right\|_{2}$.

By (5.2), (5.6-7) we obtain

$$
S_{0} \leqq \frac{1}{\pi}\|H\|\|L\| \leqq 12 \sqrt{2}\|M\|_{2}^{1 / 2}\left\|M^{+}-M^{-}\right\|_{2}^{3 / 2} \leqq 12\left\|M^{+}-M^{-}\right\|_{2}^{2},
$$

and by (5.1), (5.8) we have

$$
Q_{2} \leqq M_{0} S_{0} \leqq 12 \sqrt{2} M_{0}\|M\|_{2}^{1 / 2}\left\|M^{+}-M^{-}\right\|_{2}^{3 / 2} \leqq 12 M_{0}\left\|M^{+}-M^{-}\right\|_{2}^{2} .
$$

Now we consider the case $Q_{0}<\infty$.

Theorem 5.3. Let $k$ be SGQ and $Q_{0}<\infty$. Then

$$
\begin{gathered}
Q_{0} \leqq \frac{1}{\pi} \int \frac{u q(u) d u}{p(u)} \leqq \sum_{n \geqq 1} \frac{h_{n} L_{n}}{\pi p_{n}}, \\
Q_{0} \leqq 24\left\|M^{+}-M^{-}\right\|_{1}^{2}, \\
Q_{0} \leqq \\
\frac{128}{\pi^{2}}\left(\sum_{n \geqq 1}\left(2 p_{n}\right)\left|M_{n}^{+}-M_{n}^{-}\right|\right)^{2} .
\end{gathered}
$$

Proof. Let the integral $\pi J=\int u q(u) d u / p(u)$. By (4.2) we get $2 Q_{0}=S_{-1}$, then

$$
Q_{0}=\frac{1}{\pi} \int \sqrt{\frac{u q(u)}{p(u)}} \sqrt{\frac{p(u) q(u)}{u}} d u \leqq J^{1 / 2} Q_{0}^{1 / 2},
$$

then we get $Q_{0} \leqq J$. Hence

$$
Q_{0} \leqq \int \frac{u q(u) d u}{\pi p(u)}=\int_{0}^{\infty} \frac{q(u) d u^{2}}{\pi p(u)}=\sum_{n \geqq G_{n}} \frac{q(u) d u^{2}}{\pi p_{n}} \leqq \sum_{n \geqq 1} \frac{h_{n} L_{n}}{\pi p_{n}} .
$$

By (2.26-27) we obtain

$$
2 L_{n} h_{n} \leqq 3 \pi L_{n}^{3 / 2} \sqrt{\left|M_{n}^{ \pm}\right| 2} \leqq 3 \pi L_{n}^{3 / 2} \sqrt{M_{n}^{+}-M_{n}^{-}} \leqq 24 \pi\left(2 p_{n}\right)^{3}\left(M_{n}^{+}-M_{n}^{-}\right)^{2} .
$$

Then by (5.10) we have

$$
Q_{0} \leqq \sum_{n \geqq 1} \frac{h_{n} L_{n}}{\pi p_{n}} \leqq \sum_{n \geqq 1} 24\left(2 p_{n}\right)^{2}\left(M_{n}^{+}-M_{n}^{-}\right)^{2}=24\left\|M^{+}-M^{-}\right\|_{1}^{2} .
$$

Moreover by (5.10), (2.27) we obtain

$$
Q_{0} \leqq h_{+} \sum_{n \geqq 1} \frac{L_{n}}{\pi p_{n}} \leqq h_{+} \sum_{n \geqq 1} \frac{16 p_{n}^{2}\left|M_{n}^{+}-M_{n}^{-}\right|}{\pi p_{n}},
$$

and by $h_{+}^{2} \leqq 2 Q_{0}$ we get (5.12). 
Let us consider the case of the Hill operator $H=-d^{2} / d x^{2}+V(x)$ in $L^{2}(\mathbf{R})$. In this case $p_{n}=\pi n, n \in \mathbf{Z}$. In [MO] there are identities

$$
2 Q_{0}=\int_{0}^{1} V(x) d x, \quad 8 Q_{2}=\|V\|^{2}=\int_{0}^{1} V(x)^{2} d x,
$$

and by (4.2) we obtain

$$
S_{0}=2 Q_{2}-Q_{0}^{2}=\frac{1}{4}\left\|V_{1}\right\|^{2}=\frac{1}{4} \int_{0}^{1} V_{1}(x)^{2} d x .
$$

We begin to prove the main theorems for the Hill operator.

Proof of Theorem 2.1. By (5.5) we get (2.1) and by (5.14),(5.3),(2.1) we have (2.2).

By (5.4), (2.1) we get (2.3).

By (5.1),(5.13-14), (2.2) we get (2.4).

In [K3] there is the estimate $\|\Delta\|^{2} \leqq \pi^{2}\left\|V_{1}\right\|^{2}+8\|L\|^{2}$. Then by (2.2), (2.3) we obtain (2.5).

Now we prove the next main theorem.

Proof of Theorem 2.2. By (5.6) we have $\|L\| \leqq 4\left\|M^{+}-M^{-}\right\|_{2}$.

By (5.8) we get $\left\|V_{1}\right\| \leqq 4 \sqrt{3}\left\|M^{+}-M^{-}\right\|_{2}$.

By (5.7) we get $\|h\|_{1} \leqq 3 \pi\left\|M^{+}-M^{-}\right\|_{2}$.

In [K3] there is the estimate $\|\Delta\|^{2} \leqq \pi^{2}\left\|V_{1}\right\|^{2}+8\|L\|^{2}$. Then by $\left\|V_{1}\right\| \leqq 4 \sqrt{3}$ $\left\|M^{+}-M^{-}\right\|_{2}$ and $\|L\| \leqq 4\left\|M^{+}-M^{-}\right\|_{2}$ we obtain $\|\Delta\| \leqq 8 \pi\left\|M^{+}-M^{-}\right\|_{2}$. Hence we have (2.6).

By (5.9) we get $\|V\|^{2} \leqq 96 M_{0}\left\|M^{+}-M^{-}\right\|_{2}^{2}$ and then we get (2.7).

By $(2.26-27)$ and $p_{n}=\pi n$ we obtain (2.8-9).

By point iv) of Theorem 2.5 we obtain that $M_{0} \geqq-M_{m}^{-}$for the first nondegenerate gap $G_{m}$.

Now we remark that the proof of Theorem 2.3 follows from Theorem 3.1.

Proof of Theorem 2.3. By (2.15) and the simple equality $M_{0}+\sum_{n>0}\left(M_{n}^{+}+M_{n}^{-}\right)=$ $1 / 2$ (see for example [KK1]) we have (2.10).

By (2.16) and $Q_{0}=\sum_{n>0}\left(A_{n}^{+} M_{n}^{+}+A_{n}^{-} M_{n}^{-}\right)$(see [K2]) we have (2.11).

By $(2.17-18),(5.14)$ and $6 Q_{2}=Q_{0}+2 \sum_{n>0}\left(\left(A_{n}^{+}\right)^{2} M_{n}^{+}+\left(A_{n}^{-}\right)^{2} M_{n}^{-}\right)$(see [K2]) we have (2.12).

In [K2] there is the proof that all series converge absolutely.

Acknowledgements. The author would like to thank S. Agmon for the hospitality of Hebrew University and Y. Avron for the hospitality of Technion.

\section{References}

[F] Firsova, N.: Direct and inverse problem of scattering for one dimensional perturbed Hill operator. Mat. Sb. 130 (172), 349-385 (1986), in Russian

[GT1] Garnett, J., Trubowitz, E.: Gaps and bands of one dimensional periodic Schrödinger operator. Comment. Math. Helv. 59, 258-312 (1984) 
[GT2] Garnett, J., Trubowitz, E.: Gaps and bands of one dimensional periodic Schrödinger operator II. Comment. Math. Helv. 62, 18-37 (1987)

[J] Jenkins, A.: Univalent functions and conformal mapping. Berlin, Göttingen, Heidelberg: Springer, 1958

[KK1] Kargaev, P., Korotyaev, E.: Effective masses and conformal mappings. Commum. Math. Phys. 169, 597-625 (1995). Doklady RAN 336, 312-315 (1994) (Russian)

[KK2] Kargaev, P., Korotyaev, E.: Inverse Problem for the Hill Operator, the Direct Approach. Vienna, Preprint ESI 151, 1994, 23 pp

[KK3] Kargaev, P., Korotyaev, E.: The Inverse Problem Generated by the Conformal Mappings on the Complex Plane with Parallel Slits. To be published

[Koo] Koosis, P.: The Logarithmic Integral. Cambridge: Cambridge University Press, 1988

[K1] Korotyaev, E.: Propagation of the waves in the one-dimensional periodic media. Vienna, Preprint ESI 152, 1994, 23 pp. Doklady RAN 336, 171-174 (1994) (Russian)

[K2] Korotyaev, E.: The Second Order Estimates for the Hill Operator. Vienna, Preprint ESI $161,1994,10 \mathrm{pp}$

[K3] Korotyaev, E.: The Uniform Estimates for the Hill Operator. To be published in Doklady RAN

[K4] Korotyaev, E.: The metric properties of the conformal mapping on the complex plane with parallel slits. IMRN 10, 493-503 (1996)

[L] Levin, B.: Majorants in the class of subharmonic functions. 1-3. Theory of functions, functional analysis and their applications 51, 3-17 (1989); 52, 3-33 (1989) (in Russian)

[M] Marchenko, V.: Sturm-Liouville operator and applications. Basel: Birkhäuser, 1986

[MO] Marchenko, V., Ostrovski, I.: A characterization of the spectrum of the Hill operator. Mat. Sb. 97 (139), 540-606 (1975) (in Russian)

[Mos] Moser, J.: An Example of a Schrödinger operator with almost periodic potential and nowhere dense spectrum. Comment. Math. Helv. 56, 198-224 (1981)

[PT] Pastur, L., Tkachenko, V.: The spectral theory of some class of one dimensional Schrödinger operator with limit periodic potentials. Trudy of Moscow Math. Society 51, 114-168 (1988) (in Russian)

[S] Savin, A.: Sbornik nauchnykh trudov UFTP M.-1988 (in Russian)

Communicated by B. Simon 